\title{
Endemic angiosperms in Bahia Coastal Forests, Brazil: an update using a newly delimited area
}

\author{
Piera Ostroski ${ }^{1 *}$, Felipe Zamborlini Saiter ${ }^{2}$, André Márcio Amorim ${ }^{3,4}$ \& Pedro Fiaschi ${ }^{5}$ \\ ${ }^{1}$ Universidade Federal de Santa Catarina, Programa de Pós-Graduação em Biologia de Fungos, \\ Algas e Plantas, Florianópolis, SC, Brasil \\ ${ }^{2}$ Instituto Federal de Educação Ciência e Tecnologia do Espírito Santo, Campus Santa Teresa, \\ Santa Teresa, ES, Brasil \\ ${ }^{3}$ Universidade Estadual de Santa Cruz, Ilhéus, BA, Brasil \\ ${ }^{4}$ Comissão Executiva do Plano da Lavoura Cacaueira, Ilhéus, BA, Brasil \\ ${ }^{5}$ Universidade Federal de Santa Catarina, Departamento de Botânica, Florianópolis, SC, Brasil \\ *Corresponding author: Piera Ostroki, e-mail: piera.ostroski@gmail.com
}

OSTROSKI, P., SAITER, F. Z., AMORIM, A. M., FIASCHI, P. Endemic angiosperms in Bahia Coastal Forests, Brazil: an update using a newly delimited área. Biota Neotropica. 18(4): e20180544. http://dx.doi. org/10.1590/1676-0611-BN-2018-0544

\begin{abstract}
The forest along the coast of Bahia state, in northeastern Brazil, has been acknowledged as an area of endemism for several groups of organisms. The first study concerning endemic angiosperm species in this region, through which 395 taxa were registered, was conducted 15 years ago. However, this number is probably underrated. In this article, we present an updated checklist of angiosperm taxa in Bahia Coastal Forests (BCF) based on geographic distribution data available from the Brazilian Plant List (Flora do Brasil 2020). The records were checked in herbaria, recent taxonomic literature, and, when necessary, reviewed by experts. The final checklist consists of 547 taxa endemic to BCF, distributed in 69 families, with seven endemic genera: Bahiella (Apocynaceae), Santosia (Asteraceae), Harleyodendron (Fabaceae), Cubitanthus (Linderniaceae), Anomochloa and Parianella (Poaceae), and Andreadoxa (Rutaceae). The families with highest richness in endemic taxa were Bromeliaceae (108 spp.), Fabaceae (41 spp.) and Myrtaceae (32 spp.). This updated checklist shares only 143 taxa with a previous list published about 15 years ago. Those two lists differ mostly due to slightly different area limits, and to the large number of additional endemic species described since then. We hope our list will be used as a resource in future studies and contributes to the conservation of the highly diverse Bahia Coastal Forest.
\end{abstract}

Keywords: Atlantic Forest, checklist, conservation, endemism, hotspot.

\section{Angiospermas endêmicas da Floresta Costeira da Bahia, Brasil: uma atualização utilizando uma nova delimitação de área}

Resumo: A floresta costeira do estado da Bahia, no nordeste do Brasil, tem sido apontada como área de endemismo para diversos grupos de organismos. O primeiro levantamento de espécies de angiospermas endêmicas dessa região foi realizado há cerca de 15 anos e revelou a ocorrência de 395 táxons. Entretanto, esse número provavelmente está subestimado. Neste trabalho apresentamos uma lista atualizada de táxons de angiospermas endêmicas da Floresta Costeira da Bahia (FCB), tendo como base as informações de distribuição geográfica disponíveis na lista da Flora do Brasil. Estas informações foram posteriormente checadas em registros de herbários, estudos taxonômicos recentes e, quando necessário, revisadas por especialistas. A lista final de espécies é constituída por 547 táxons endêmicos da Floresta Costeira da Bahia, distribuídos em 69 famílias e incluindo sete gêneros endêmicos: Bahiella (Apocynaceae), Santosia (Asteraceae), Harleyodendron (Fabaceae), Cubitanthus (Linderniaceae), Anomochloa $\mathrm{e}$ Parianella (Poaceae), and Andreadoxa (Rutaceae). As famílias mais ricas em táxons endêmicos são Bromeliaceae (108 spp.), Fabaceae (41 spp.) e Myrtaceae (32 spp.). Apenas 143 táxons são compartilhados por esta lista atualizada e uma lista preliminar publicada há cerca de 15 anos. As duas listas diferem especialmente por ligeiras diferenças nos limites da área de estudo e também pelo acréscimo de novas espécies endêmicas descritas desde então. Espera-se que a lista seja usada como ferramenta para estudos futuros e para a conservação da rica flora costeira da Bahia.

Palavras-chave: Checklist, conservação, endemismo, hotspot, Mata Atlântica. 


\section{Introduction}

The Atlantic Forest is considered a biodiversity hotspot due to the occurrence of a high number of endemic and threatened species (Myers et al. 2000). The natural distribution of the Atlantic Forest was originally continuous along the Brazilian coast and extended to some areas in Argentina and Paraguay (Fundação SOS Mata Atlântica 2013). Nowadays, it is one of the eight most critically endangered hotspots, as only $12,5 \%$ of the original forest remains (Fundação SOS Mata Atlântica 2014). Nevertheless, it is the richest Brazilian phytogeographic domain in plant diversity, with 8,728 endemic species of angiosperms (Flora do Brasil 2020, under construction).

Some studies have emphasized the division of the Atlantic Forest in northern and southern regions (Cracraft 1985, Colombi et al. 2010, Martins 2011) with a limit along the Doce river, in the north of Espírito Santo state. The floristic differences between these two regions were highlighted by Oliveira-Filho \& Fontes (2000) and Oliveira-Filho et al. (2005), who observed several taxa with Amazonian affinities occuring in the northern region, while taxa with subtropical Andean affinities are mostly found in the southern region (Berry et al. 2004, Santos et al. 2007, Menini Neto et al. 2016). Other authors recognize three main centers of endemism in the Atlantic Forest: northern (Pernambuco and Alagoas states), southern (from Rio de Janeiro to Santa Catarina states), and central (southern Bahia and Espírito Santo states) (Murray-Smith et al. 2009, Thomas et al. 1998).

Recently, Saiter et al. (2016a) divided the central region of the Atlantic Forest in three subregions based on the composition of tree species, indicating the most important abiotic factors affecting that division. Contrary to previous knowledge, the rivers did not play an important role in this division, which lies about $100 \mathrm{~km}$ north of the Doce river and is best explained by climatic factors, such as moisture, elevation and temperature (Saiter et al. 2016a). One of the regions, referred to as Bahia Coastal Forests (BCF) by Saiter et al. (2016a), encompasses the northern extreme of Espírito Santo and most of the coast of the state of Bahia. Due to high biodiversity and the incidence of endemic and threatened species, this region has been designated as a hot-point within the Atlantic Forest hotspot (Martini et al. 2007). Many studies to this date on different groups of organisms support this claim, as the region is considered an area of endemism for frogs (Carnaval et al. 2009), birds (Silva et al. 2004), Myrtaceae (Murray-Smith et al. 2009) and trees in general (Thomas et al. 2003).

An estimate of endemic vascular plant species present in two protected areas in southern Bahia showed that the distribution of $59 \%$ of the species is restricted to Bahia and Espírito Santo states (Thomas et al. 1998). These results motivated the compilation of a preliminary list of endemic angiosperm species in the region comprising Espírito Santo to the north of the Doce river and the south of Bahia, including inland Seasonal Dry Forests (Thomas et al. 2003) (Figure 1a). This list consisted of 395 endemic species of angiosperms in the region (Thomas et al. 2003), among which several are known to occur only in dry forests, such as Chrysophyllum subspinosum Monach. (Sapotaceae) and Colicodendron bahianum Cornejo \& Ilts (Capparaceae). That study reported eight endemic genera in this area: Arapatiella R.S.Cowan, Brodriguesia R.S.Cowan and Harleyodendron R.S.Cowan (Fabaceae), Atractantha McClure, Anomochloa Brogn., Alvimia Calderón ex Soderstr. \& Londoño, and Sucrea Soderstr.
(Poaceae), and Trigoniodendron E.F.Guim \& Miguel (Trigoniaceae) (Thomas et al, 2003).

Checklists of endemic taxa are critical to overcome issues that hinder conservation strategies in highly biodiverse regions, such as: 1) basic taxonomic information (Linnean shortfall, or "which species are there?"), and 2) lack of geographic information (Wallacean shortfall, or “where do these species occur?") (Whittaker et al. 2005). Both shortfalls are part of reality in Bahia Coastal Forests, world-renowned as one of the highest diversity areas in tree species (Thomas et al. 1998, Martini et al. 2007, Murray-Smith et al. 2009).

We aimed to provide a list of endemic species of angiosperms in Bahia Coastal Forest following Saiter et al. (2016a) to verify whether the current list agrees with the preliminary checklist by Thomas et al. (2003). We expect this updated list to further highlight the importance of BCF as a critical area for biodiversity conservation in the Brazilian Atlantic forest.

\section{Material and Methods}

\section{Study area}

To delimit the study area we used the BCF sensu Saiter et al. (2016a) (Figure 1b) with a slight modification to include the municipalities whose territory was at least $50 \%$ inside that perimeter. Municipalities were used as a base for the retrieval of taxonomic information from online datasets (see data collection). To remove the arbitrary northern limit from Saiter et al. (2016a) (Figure 1b), the study area was expanded to include seven municipalities complying with the $50 \%$ territory criterion (Figure 1c). Municipal limits were defined based on data from the Brazilian Institute for Geography and Statistics (IBGE 1993) (Figure 1c).

The resulting area comprised 156 municipalities, which account for approximately $99,000 \mathrm{~km}^{2}$ (Figure 1c) at southern Bahia state, and a small fraction from northern Espírito Santo state and northeastern Minas Gerais state, contrasting with an area of about $119,000 \mathrm{~km}^{2}$ at southern Bahia which included more inland areas, and northen Espírito Santo with a southern limit at the Doce River (Thomas et al. 2003) (Figure 1a).

\section{Data collection}

To compile the checklist of endemic species of angiosperms in BCF, we started by performing a data search in the Brazilian Plant List (Flora do Brasil 2020) on March 9, 2017, using the following filters simultaneously: "group: angiosperms", "endemism: only endemic to Brazil", "origin: native", "state: Bahia", and "phytogeographic domain: Atlantic Forest". We decided not to search only for species endemic to the Atlantic Forest in Bahia to avoid excluding species that could also occur in the north of Espírito Santo and in a small area in northeastern Minas Gerais (Figure 1c). The initial search resulted in 3,338 species, 59 subspecies and 154 varieties (3,551 taxa). These records were then checked in the SpeciesLink database (splink.cria.org.br). Each taxon map generated by the SpeciesLink website was visually checked, first to remove taxa that were clearly not endemic to BCF, i.e. those with several records outside the study area. Each of the 1,336 remaining taxa were then mapped with QGis 2.14 (Quantum GIS Development Team 2016) and we verified whether the points of occurrence were enclosed in the limits of the study area, which resulted in the further removal of 701 taxa. 


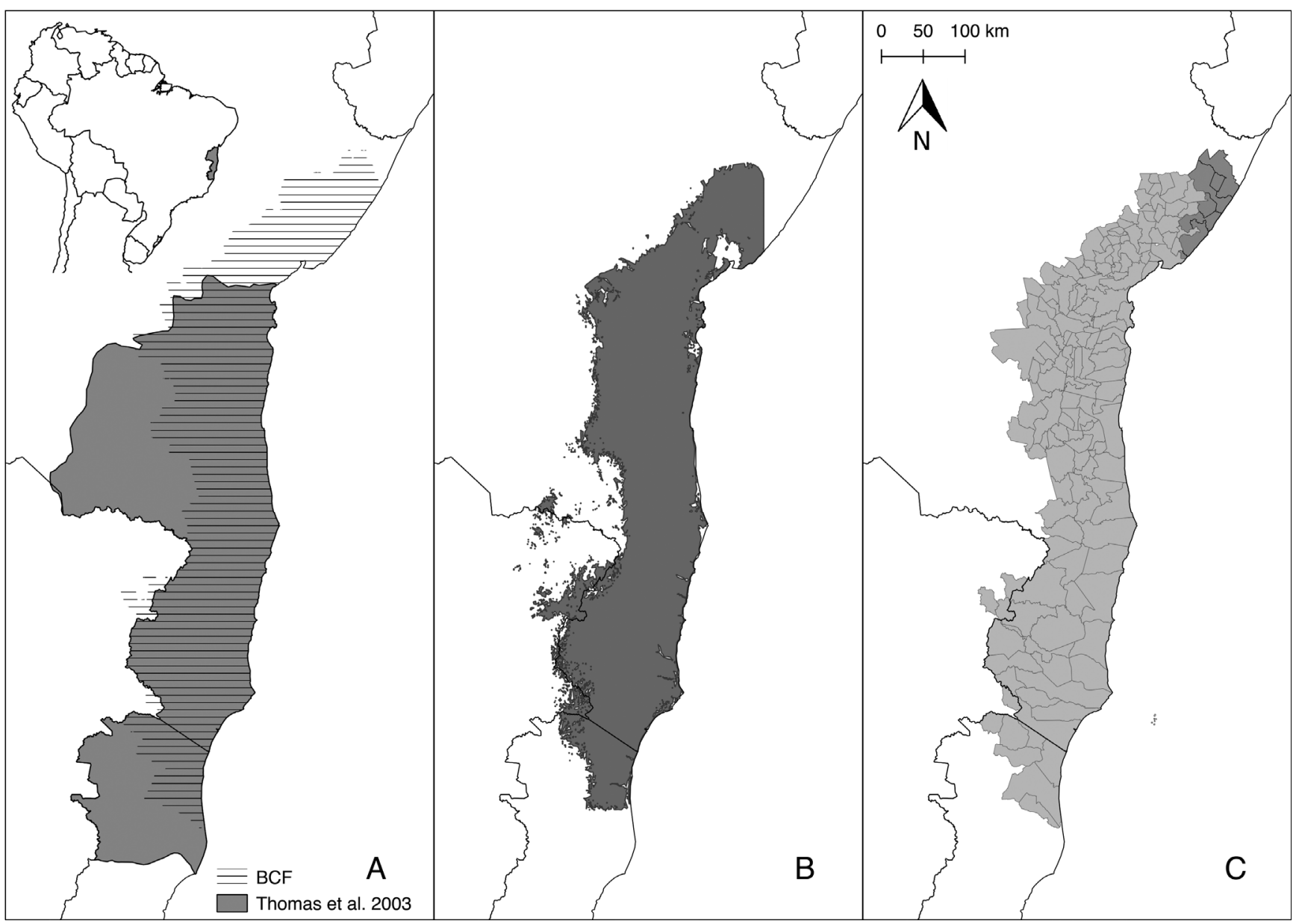

Figure 1. a) Study area in which Thomas et al. (2003) based their list of endemic species. b) Bahia Coastal Forest (BCF) according to Saiter et al. (2016a). c) Municipalities with at least $50 \%$ of its area within the BCF (light grey), and those added to the study area based on IBGE (1993) (dark grey). Coordinates: UTM. Datum WGS 84.

From the 635 taxa left, the records with incongruent data were verified by experts or checked using recent taxonomic studies (Appendix 1). This step resulted in 30 additional non-endemic species discarded from the list. The taxa for which no scientific records were found in Google Scholar (scholar.google.com), Biodiversity Heritage Library (biodiversitylibrary.com), SpeciesLink (splink.cria.org.br), and the Brazilian Plant List (Flora do Brasil 2020) were complemented by a search for location records in the CEPEC herbarium. Once this verification was completed, 53 species from the list had to be removed because no records were available and a further five species were removed due to lack of precise geographic information.

\section{Results}

The final checklist consisted of 547 angiosperm taxa endemic to $\mathrm{BCF}$ ( 535 species, six subspecies, and six varieties) in 69 families (none endemic) and 230 genera (Table 1). Seven genera are endemic to BCF: Bahiella J.F.Morales (Apocynaceae), Santosia R.M.King \& H.Rob. (Asteraceae), Harleyodendron (Fabaceae, Figure 2f), Cubitanthus Barringer (Linderniaceae), Anomochloa and Parianella Hollowell, F.M.Ferreira \& R.P.Oliveira (Poaceae), and Andreadoxa Kallunki (Rutaceae). The genera with the highest number of endemic species in the BCF were Aechmea Ruiz \& Pav. (Bromeliaceae) (33 spp.),
Pavonia Cav. (Malvaceae) and Myrcia DC. (Myrtaceae), each with 16 species. The three families with the highest numbers of endemic taxa (species + infraspecific taxa) were Bromeliaceae (108), Fabaceae (41) and Myrtaceae (32).

\section{Discussion}

The families with the highest numbers of endemic taxa in BCF are also among the ten richest angiosperm families in Brazil and in the Atlantic Forest (BFG 2015). Thomas et al. (2003) reported Fabaceae (53 spp.) as the richest family of a total of 65 families in the previous list. Compared with the BFG (2015), it is remarkable that Orchidaceae, one of the three richest families in Brazil and in the Atlantic Forest, was not among the richest families in this study, with only 25 taxa. This may be due to the lack of geographic records for 14 species in this family, which had to be discarded.

Among the 20 richest angiosperm genera in Brazil (BFG 2015), Aechmea and Myrcia are also among the genera with the highest number of endemic species in the BCF. In a preliminary list of the endemic angiosperm species from southern Bahia and northern Espírito Santo, Thomas et al. (2003) cited Conchocarpus J.C.Mikan (Rutaceae) (19 spp.) as the richest genus, followed by Pavonia Cav. (Malvaceae) (13 spp.), Couepia Aubl., and Erythroxylum P.Browne (seven spp. each). 
Ostroski, P. et al.

Table 1. Checklist of angiosperms endemic to Bahia Coastal Forest sensu Saiter et al. (2016a). * Taxon also in Thomas et al. (2003).

\author{
Acanthaceae \\ Aphelandra ignea Nees ex Steud. \\ Clistax bahiensis Profice \& Leitman \\ Harpochilus phaeocarpus Nees \\ Herpetacanthus magnobracteolatus Indriunas \& Kameyama \\ Herpetacanthus strongyloides Indriunas \& Kameyama \\ Herpetacanthus tetrandrus (Nees \& Mart.) Herter \\ Justicia antirrhina Nees \& Mart. \\ Justicia physogaster Lindau \\ Lepidagathis cuneiformis Kameyama \\ Mendoncia bahiensis Profice \\ Mendoncia blanchetiana Profice \\ Pseuderanthemum albiflorum (Hook.) Radlk. \\ Pseuderanthemum verbenaceum (Nees \& Mart.) Radlk. \\ Ruellia sessilifolia (Nees) Lindau \\ Schaueria gonyostachya (Nees \& Mart.) Nees \\ Schaueria hirsuta Nees \\ Schaueria marginata Nees \\ Schaueria pyramidalis A.L.A.Côrtes
}

\section{Achariaceae}

Kuhlmanniodendron macrocarpum Groppo, Favaretto \& Fiaschi

\section{Amaranthaceae}

Lecosia formicarum Pedersen

\section{Amaryllidaceae}

Griffinia arifolia Ravenna

Griffinia parviflora Ker Gawl.

Griffinia paubrasilica Ravenna

\section{Annonaceae}

Annona bahiensis (Maas \& Westra) H.Rainer *

Duguetia magnolioidea Maas *

Duguetia restingae Maas *

Duguetia reticulata Maas *

Guatteria stenocarpa Lobão, Maas \& Mello-Silva

Hornschuchia cauliflora Maas \& Setten *

Hornschuchia leptandra D.M.Johnson *

Hornschuchia obliqua Maas \& Setten *

Hornschuchia polyantha Maas *

Hornschuchia santosii D.M.Johnson *

Malmea obovata R.E.Fr. *

Pseudoxandra bahiensis Maas *

Unonopsis bahiensis Maas \& Orava

Xylopia involucrata M.C.Dias \& Kinoshita *

\section{Apocynaceae}

Aspidosperma thomasii Marc.-Ferr. *

Bahiella blanchetii (A.DC.) J.F.Morales

Bahiella infundibuliflora J.F.Morales
Continuation Table 1.

Lacmellea bahiensis J.F.Morales

Marsdenia carvalhoi Morillo \& Carnevali

Matelea riparia Morillo

Matelea santosii Morillo \& Fontella

Oxypetalum laciniatum Rapini \& Farinaccio

Rauvolfia atlantica Emygdio

\section{Araceae}

Anthurium bromelicola subsp. bahiense Mayo et al. *

Anthurium illepidum Schott

Anthurium molle E.G.Gonç. \& J.G.Jardim

Anthurium teimosoanum E.G.Gonç. \& J.G.Jardim

Asterostigma riedelianum (Schott) Kuntze

Dracontioides salvianii E.G.Gonç.

Philodendron aemulum Schott

Zomicarpa steigeriana Maxim. ex Schott

Araliaceae

Schefflera aurata Fiaschi

\section{Arecaceae}

Bactris soeiroana Noblick ex A.J.Hend.

Geonoma pohliana subsp. rubescens (Wendland ex Drude)

Henderson

Geonoma pohliana subsp. unaensis Henderson

Syagrus $\times$ camposportoana (Bondar) Glassman

Syagrus itapebiensis (Noblick \& Lorenzi) Noblick \& Meerow

Syagrus santosii K. Soares \& C.A.Guim.

\section{Aristolochiaceae}

Aristolochia brunneomaculata I.Abreu \& Giul.

Aristolochia longispathulata F.González *

\section{Asparagaceae}

Hagenbachia brasiliensis Nees \& Mart.

\section{Asteraceae}

Acmella paniculata (Wall. ex DC.) R.K.Jansen

Austroeupatorium morii R.M.King \& H.Rob.

Barrosoa atlantica R.M.King \& H.Rob. *

Diacranthera hebeclinia H.Rob.

Litothamnus ellipticus R.M.King \& H.Rob. *

Mikania amorimii Borges \& Forzza

Mikania kubitzkii R.M.King \& H.Rob. *

Mikania mattos-silvae R.M.King \& H.Rob. *

Mikania santosii R.M.King \& H.Rob. *

Piptocarpha riedelii (Sch.Bip.) Baker

Santosia talmonii R.M.King \& H.Rob. *

Vernonanthura vinhae (H.Rob.) H.Rob. *

Begoniaceae

Begonia delicata Gregório \& J.A.S.Costa

Begonia elianeae Gregório \& J.A.S.Costa

Begonia epibaterium Mart. ex A.DC. 
Continuation Table 1.

Begonia goldingiana L.Kollmann \& A.P.Fontana Begonia mattos-silvae L.B.Sm. ex S.F.Sm. \& Wassh. * Begonia pinheironis L.B.Sm. ex S.F.Sm. \& Wassh. * Begonia russelliana L.B.Sm. ex S.F.Sm. \& Wassh. * Begonia saxifraga A.DC.

Begonia subacida Irmsch.

Begonia sylvatica Meisn. ex A.DC.

Bignoniaceae

Handroanthus parviflorus Espírito-Santo \& M.M. Silva-Castro

\section{Bromeliaceae}

Aechmea amicorum B.R.Silva \& H.Luther

Aechmea amorimii Leme *

Aechmea ampla L.B.Sm.

Aechmea andersoniana Leme \& H.Luther

Aechmea andersonii H.Luther \& Leme

Aechmea carvalhoi E.Pereira \& Leme

Aechmea correia-araujoi E.Pereira \& Moutinho

Aechmea curranii (L.B.Sm.) L.B.Sm. \& M.A.Spencer

Aechmea digitata L.B.Sm. \& R.W.Read

Aechmea discordiae Leme

Aechmea disjuncta (L.B.Sm.) Leme \& J.A.Siqueira

Aechmea echinata (Leme) Leme *

Aechmea farinosa (Regel) L.B.Sm.

Aechmea glandulosa Leme

Aechmea gregaria Leme \& L.Kollmann

Aechmea guaratingensis Leme \& L.Kollmann

Aechmea heterosepala Leme

Aechmea incompta Leme \& H.Luther

Aechmea ituberaensis Leme \& L.Kollmann

Aechmea laevigata Leme

Aechmea lanata (L.B.Sm.) L.B.Sm. \& M.A.Spencer

Aechmea limae Leme

Aechmea lymanii W.Weber

Aechmea miniata Beer ex Baker

Aechmea mira Leme \& H.Luther

Aechmea mollis L.B.Sm.

Aechmea pendulispica Leme \& L.Kollmann

Aechmea ramusculosa Leme

Aechmea tentaculifera Leme, Amorim \& J.A. Siqueira

Aechmea turbinocalyx $\mathrm{Mez}$

Aechmea viridipetala A.F.Costa \& Amorim

Aechmea viridostigma Leme \& H.Luther

Aechmea weberi (E.Pereira \& Leme) Leme

Alcantarea cerosa Leme, A.P.Fontana \& O.A.B.Ribeiro

Alcantarea pataxoana Versieux

Araeococcus montanus Leme
Continuation Table 1.

Araeococcus nigropurpureus Leme \& J.A.Siqueira

Araeococcus sessiliflorus Leme \& J.A.Siqueira

Billbergia fosteriana L.B.Sm.

Billbergia macrocalyx Hook.

Canistrum fosterianum L.B.Sm.

Canistrum guzmanioides Leme

Canistrum lanigerum H.Luther \& Leme

Canistrum sandrae Leme

Canistrum seidelianum W.Weber

Cryptanthus colnagoi Rauh \& Leme

Cryptanthus coriaceus Leme

Cryptanthus ilhanus Leme

Cryptanthus lyman-smithii Leme

Cryptanthus pseudopetiolatus Philcox *

Cryptanthus ruthiae Philcox

Cryptanthus ubairensis I.Ramírez

Cryptanthus vexatus Leme

Cryptanthus viridovinosus Leme

Cryptanthus walkerianus Leme \& L.Kollmann

Hohenbergia barbarespina Leme \& Fraga

Hohenbergia brachycephala L.B.Sm.

Hohenbergia burle-marxii Leme \& W.Till

Hohenbergia capitata Schult. \& Schult.f.

Hohenbergia castellanosii L.B.Sm. \& Read

Hohenbergia correia-araujoi E.Pereira \& Moutinho

Hohenbergia flava Leme \& C.C.Paula

Hohenbergia hatschbachii Leme *

Hohenbergia itamarajuensis Leme \& Baracho

Hohenbergia littoralis L.B.Sm.

Hohenbergia pabstii L.B.Sm. \& Read

Hohenbergia reconcavensis Leme \& Fraga

Hohenbergia sandrae Leme

Lymania alvimii (L.B.Sm. \& R.W.Read) R.W.Read *

Lymania azurea Leme

Lymania brachycaulis (E.Morren ex Baker) L.F.Sousa

Lymania corallina (Brong. ex Beer) R.W.Read

Lymania globosa Leme

Lymania languida Leme

Lymania marantoides (L.B.Sm.) R.W.Read

Lymania spiculata Leme \& Forzza *

Neoregelia azevedoi Leme

Neoregelia crispata Leme*

Neoregelia longisepala E.Pereira \& I.A.Penna *

Neoregelia rothinessa Leme, H.Luther \& W.Till

Neoregelia silvomontana Leme \& J.A.Siqueira

Neoregelia viridolineata Leme 
Ostroski, P. et al.

Continuation Table 1.

Neoregelia wilsoniana M.B.Foster

Orthophytum buranhense Leme \& A.P.Fontana

Orthophytum guaratingense Leme \& L.Kollmann

Orthophytum rubrum L.B.Sm.

Portea alatisepala Philcox *

Portea filifera L.B.Sm.

Portea grandiflora Philcox *

Portea kermesina K.Koch

Portea nana Leme \& H.Luther

Quesnelia alborosea A.F.Costa \& T.Fontoura

Quesnelia clavata Amorim \& Leme

Quesnelia dubia Leme

Quesnelia koltesii Amorim \& Leme

Ronnbergia brasiliensis E.Pereira \& I.A.Penna *

Ronnbergia carvalhoi Martinelli \& Leme*

Ronnbergia neoregelioides Leme

Ronnbergia silvana Leme

Vriesea dictyographa Leme

Vriesea graciliscapa W.Weber

Vriesea longisepala A.F.Costa

Vriesea minuta Leme

Vriesea minutiflora Leme

Vriesea roberto-seidelii W.Weber

Vriesea ruschii L.B.Sm. subsp. ruschii

Vriesea sandrae Leme

Vriesea silvana Leme

\section{Burseraceae}

Protium icicariba var. talmonii Daly *

Trattinnickia lorenziana Daly \& M.F.F.Melo

\section{Cactaceae}

Rhipsalis hileiabaiana (N.P.Taylor \& Barthlott) N.Korotkova \& Barthlott *

\section{Calophyllaceae}

Kielmeyera itacarensis Saddi

Kielmeyera marauensis Saddi

\section{Capparaceae}

Colicodendron martianum Cornejo

\section{Caryocaraceae}

Caryocar edule Casar.

\section{Chrysobalanaceae}

Couepia bondarii Prance *

Couepia coarctata Prance *

Couepia impressa subsp. cabraliae Prance *

Couepia longipetiolata Prance *

Licania bahiensis Prance

Licania lamentanda Prance *
Continuation Table 1.

Licania littoralis Warm. var. littoralis

Licania santosii Prance *

Licania turbinata Benth.

Parinari alvimii Prance *

\section{Clusiaceae}

Tovomita iaspidis L.Marinho \& Amorim

Tovomita megantha L.Marinho \& Amorim

Commelinaceae

Dichorisandra jardimii Aona \& M.C.E.Amaral

Dichorisandra leucophthalmos Hook.

Dichorisandra leucosepala Aona \& M.C.E.Amaral

Dichorisandra marantoides Aona \& Faden

Dichorisandra ordinatiflora Aona \& Faden

Dichorisandra radicalis Nees \& Mart.

Dichorisandra subtilis Aona \& M.C.E.Amaral

\section{Connaraceae}

Connarus blanchetii var. laurifolius (Baker) Forero

Connarus cuneifolius Baker

Connarus portosegurensis Forero

Rourea bahiensis Forero *

Rourea carvalhoi Forero et al. *

Rourea discolor Baker

Rourea macrocalyx Carbonó et al. *

Rourea tenuis G.Schellenb.

\section{Cucurbitaceae}

Cayaponia nitida Gomes-Klein \& Pirani

Fevillea bahiensis G.Rob. \& Wunderlin

Gurania wawrei Cogn.

\section{Cyclanthaceae}

Asplundia maximiliani Harling

Cyperaceae

Becquerelia discolor Kunth

Hypolytrum bahiense M.Alves \& W.W.Thomas *

Hypolytrum jardimii M.Alves \& W.W.Thomas *

Hypolytrum lucennoi M.Alves \& W.W.Thomas

\section{Dichapetalaceae}

Stephanopodium magnifolium Prance *

Tapura martiniae Amorim \& D.Lisboa

Tapura zei-limae Amorim \& Fiaschi

Dilleniaceae

Davilla bahiana Aymard

Davilla macrocarpa Eichler

Dioscoreaceae

Dioscorea macrothyrsa Uline

Ebenaceae

Diospyros amabi B.Walln. 
Continuation Table 1.

Diospyros riedelii (Hiern) B.Walln.

Diospyros scottmorii B.Walln.

\section{Eriocaulaceae}

Actinocephalus ochrocephalus (Körn.) Sano

\section{Erythroxylaceae}

Erythroxylum compressum Peyr.

Erythroxylum leal-costae Plowman

Erythroxylum martii Peyr.

Erythroxylum mattos-silvae Plowman *

Erythroxylum membranaceum Plowman *

Erythroxylum santosii Plowman *

Erythroxylum splendidum Plowman*

\section{Euphorbiaceae}

Actinostemon lasiocarpus (Müll.Arg.) Baill.

Algernonia bahiensis (Emmerich) G.L.Webster *

Bernardia gambosa Müll.Arg.

Bernardia micrantha Pax \& K.Hoffm.

Croton sapiifolius Müll.Arg.

Croton thomasii Riina \& P.E.Berry

Dalechampia armbrusteri G.L.Webster

Dalechampia viridissima G.L.Webster*

Gymnanthes gaudichaudii Müll.Arg.

Ophthalmoblapton pedunculare Müll.Arg.

\section{Fabaceae}

Andira carvalhoi R.T.Penn. \& H.C.Lima*

Andira marauensis N.F.Mattos *

Arapatiella psilophylla (Harms) R.S.Cowan *

Canavalia cassidea G.P.Lewis *

Canavalia dolichothyrsa G.P.Lewis *

Chamaecrista amabilis H.S.Irwin \& Barneby *

Chamaecrista amorimii Barneby *

Chamaecrista onusta H.S.Irwin \& Barneby *

Chamaecrista salvatoris (H.S.Irwin \& Barneby) H.S.Irwin \& Barneby

Copaifera majorina Dwyer

Dahlstedtia bahiana (A.M.G.Azevedo) M.J.Silva \&

A.M.G.Azevedo

Harleyodendron unifoliolatum R.S.Cowan *

Inga aptera (Vinha) T.D.Penn. *

Inga conchifolia L.P.Queiroz

Inga pedunculata (Vinha) T.D.Penn. *

Inga pleiogyna T.D.Penn.

Machaerium aureum Filardi \& H.C.Lima

Moldenhawera blanchetiana var. multijuga L.P.Queiroz et al.

Moldenhawera intermedia G.P.Lewis \& L.P.Queiroz

Moldenhawera luschnathiana Yakovlev

Moldenhawera nutans L.P.Queiroz et al.
Continuation Table 1.

Muellera longiunguiculata (MJ.Silva \& AMG.Azevedo) MJ.Silva \& AMG.Azevedo

Ormosia lewisii D.B.O.S.Cardoso, C.H.Stirt. \& Torke *

Ormosia limae D.B.O.S.Cardoso \& L.P.Queiroz

Ormosia timboensis D.B.O.S.Cardoso, Meireles \& H.C.Lima

Parapiptadenia ilheusana G.P.Lewis *

Phanera carvalhoi (Vaz) Vaz

Piptadenia killipii var. cacaophila G.P.Lewis *

Piptadenia ramosissima Benth.

Piptadenia santosii Barneby ex G.P.Lewis *

Schnella lilacina (Wunderlin \& Eilers) Wunderlin

Senegalia amorimii M.J.F.Barros \& M.P.Morim

Senegalia olivensana (G.P.Lewis) Seigler \& Ebinger

Senegalia piptadenioides (G.P.Lewis) Seigler \& Ebinger

Swartzia alternifoliolata Mansano

Swartzia arenophila R.B.Pinto, Torke \& Mansano

Swartzia curranii R.S.Cowan

Swartzia pinheiroana R.S.Cowan *

Swartzia riedelii R.S.Cowan

Swartzia thomasii R.B.Pinto, Torke \& Mansano

Zollernia magnifica A.M.Carvalho \& Barneby *

Gentianaceae

Macrocarpaea atlantica J.R.Grant \& V.Trunz

Macrocarpaea dolichophylla J.R.Grant \& V.Trunz

Macrocarpaea orbiculata J.R.Grant \& V.Trunz

Icacinaceae

Pleurisanthes brasiliensis (Val.) Tiegh.

Iridaceae

Neomarica brachypus (Baker) Sprague

Neomarica floscella A.Gil \& M.C.E.Amaral

Neomarica portosecurensis (Ravenna) Chukr *

Neomarica unca (Ravenna) A.Gil

Lauraceae

Nectandra micranthera Rohwer

Ocotea montana (Meisn.) Mez

Ocotea ramosissima L.C.S.Assis e Mello-Silva

Ocotea sperata P.L.R.Moraes et van der Werff

Ocotea thinicola van der Werff et P.L.R.Moraes

Lecythidaceae

Eschweilera complanata S.A.Mori

Eschweilera mattos-silvae S.A.Mori *

Eschweilera sphaerocarpa M.Ribeiro \& S.A.Mori

Linderniaceae

Cubitanthus alatus (Cham. \& Schltdl.) Barringer

\section{Loganiaceae}

Spigelia genuflexa Popovkin \& Struwe

Strychnos alvimiana Krukoff \& Barneby * 
Ostroski, P. et al.

Continuation Table 1.

Strychnos setosa Krukoff \& Barneby

Loranthaceae

Psittacanthus excrenulatus Rizzini

Psittacanthus salvadorensis Kuijt

Struthanthus longiflorus Rizzini

\section{Malpighiaceae}

Bunchosia itacarensis W.R.Anderson *

Heteropterys sanctorum W.R.Anderson *

Peixotoa sericea C.E.Anderson *

Stigmaphyllon hispidum C.E.Anderson

Stigmaphyllon macropodum A.Juss.

\section{Malvaceae}

Byttneria cristobaliana Dorr

Pavonia cauliflora (Nees) Fryxell ex G.L.Esteves

Pavonia ciliata G.L.Esteves \& Krapov. *

Pavonia crispa Krapov. *

Pavonia gerleniae Gonçalez \& M.C.Duarte

Pavonia goetheoides (Hassl.) Fryxell ex G.L.Esteves

Pavonia latibracteolata Krapov. *

Pavonia longifolia A.St.-Hil.

Pavonia macrobracteolata Gonçalez \& M.C.Duarte

Pavonia ovaliphylla G.L.Esteves \& Krapov. *

Pavonia paucidentata Fryxell

Pavonia pilifera Krapov.

Pavonia rubriphylla G.L.Esteves

Pavonia sancti Krapov.

Pavonia spectabilis Krapov. *

Pavonia spiciformis Krapov. *

Pavonia stipularis Krapov. *

\section{Marantaceae}

Goeppertia fasciata (Linden ex K.Koch) Borchs. \& S.Suárez

Goeppertia rufibarba (Fenzl) Borchs. \& S.Suárez

Ischnosiphon bahiensis L.Andersson *

Monotagma grallatum Hagberg \& R. Erikss. *

Stromanthe bahiensis Yosh.-Arns, Mayo \& J.M.A. Braga

\section{Marcgraviaceae}

Schwartzia geniculatiflora Gir.-Cañas \& Fiaschi

Melastomataceae

Bertolonia alternifolia Baumgratz, Amorim \& A.B.Jardim

Bertolonia bullata Baumgratz, Amorim \& A.B.Jardim

Huberia carvalhoi Baumgratz

Huberia sessilifolia R. Godenberg \& Michelangelo

Meriania inflata Michelangeli \& R.Goldenb.

Miconia lurida Cogn.

Ossaea loligomorpha R.Goldenb. \& Reginato

Ossaea sulbahiensis D'El Rei Souza
Continuation Table 1.

Physeterostemon aonae Amorim, Michelangeli \& R.Goldenb.

Physeterostemon fiaschii R.Goldenb. \& Amorim

Physeterostemon jardimii R.Goldenb. \& Amorim

Physeterostemon thomasii Amorim, Michelangeli \& R.Goldenb.

Pleiochiton amorimii Reginato \& R.Goldenb.

Tibouchina bahiensis Wurdack *

Tibouchina bradeana Renner

Tibouchina paulo-alvinii Guimarães da Vinha *

Tibouchina stipulacea Vinha*

Tibouchina taperoensis Wurdack *

Tibouchina tomentulosa Wurdack

Meliaceae

Guarea anomala T.D.Penn

Trichilia florbranca T.D.Penn. *

Trichilia magnifoliola T.D.Penn. *

Menispermaceae

Curarea crassa Barneby*

\section{Moraceae}

Dorstenia setosa Moric

Myrtaceae

Calyptranthes blanchetiana O.Berg

Eugenia barrana Sobral

Eugenia beruttii (Mattos) Mattos

Eugenia fissurata Mattos

Eugenia itacarensis Mattos *

Eugenia longifolia DC.

Eugenia serraegrandis Sobral

Eugenia sessilifolia DC.

Eugenia unana Sobral

Marlierea lealcostae G.M.Barroso \& Peixoto

Marlierea verticillaris O.Berg

Myrcia abrantea (O.Berg) E.Lucas \& Sobral

Myrcia cataphyllata M.F.Santos

Myrcia felisbertii (DC.) O.Berg

Myrcia gigantea (O.Berg) Nied.

Myrcia grazielae NicLugh.

Myrcia marianae Staggemeier \& E.Lucas

Myrcia monoclada Sobral

Myrcia pendula Sobral

Myrcia pseudomarlierea Sobral

Myrcia raminfinita L.Marinho \& E.Lucas

Myrcia spathulifolia Proença

Myrcia stigmatosa O.Berg

Myrcia teimosa Sobral

Myrcia tetraphylla Sobral

Myrcia thomasii B.S.Amorim \& A.R.Lourenço 
Continuation Table 1.

Myrcia truncata Sobral
Plinia callosa Sobral *
Plinia longiacuminata Sobral
Plinia muricata Sobral *
Plinia rara Sobral *
Plinia spiciflora (Nees \& Mart.) Sobral

\section{Nyctaginaceae}

Neea alumnorum M.Pignal, Soares Filho \& Romaniuc

Neea duckei (Huber) Furlan

\section{Ochnaceae}

Ouratea bahiensis Sastre *

Ouratea gigantophylla (Erhard) Engl.

Ouratea longipes Sastre *

Ouratea papulosa Sastre

Ouratea platicaulis Sastre *

\section{Orchidaceae}

Anathallis velvetina Luer \& Toscano

Aspasia silvana F.Barros *

Bifrenaria silvana V.P.Castro *

Brassavola reginae Pabst

Bulbophyllum teimosense E.C.Smidt \& Borba

Cattleya alaorii (Brieger \& Bicalho) Van den Berg

Cattleya grandis (Lindl. \& Paxton) A.A.Chadwick

Cattleya kerrii Brieger \& Bicalho*

Cirrhaea silvana V.P.Castro \& Campacci *

Coryanthes bahiensis Marçal \& Chiron

Coryanthes bueraremensis Campacci \& Bohnke

Elleanthus hymenophorus (Rchb.f.) Rchb.f.

Encyclia fimbriata C.A.Bastos, Van den Berg \& Meneguzzo

Epidendrum garciae Pabst

Gomesa silvana (V.P.Castro \& Campacci) M.W.Chase \&

N.H.Williams

Gongora meneziana V.P.Castro \& G.Gerlach

Koellensteinia abaetana L.P.Queiroz

Leptotes bohnkiana Campacci

Masdevallia sururuana Campacci

Pabstiella dasilvae Chiron \& Xim.Bols.

Promenaea silvana F.Barros \& Cath. *

Prosthechea bohnkiana V.P.Castro \& G.F.Carr

Prosthechea bueraremensis (Campacci) Campacci

Stanhopea bueraremensis Campacci \& Marçal

Stenia bohnkiana V.P.Castro \& G.F.Carr

\section{Oxalidaceae}

Oxalis alata var. hirta Lourteig *

Oxalis bela-vitoriae Lourteig *

Oxalis kuhlmannii var. adpressipila Lourteig
Continuation Table 1.

\section{Passifloraceae}

Passiflora igrapiunensis T.S.Nunes \& L.P.Queiroz

Phyllanthaceae

Discocarpus pedicellatus Fiaschi \& Cordeiro

Phyllanthus carvalhoi G.L.Webster

\section{Picramniaceae}

Picramnia coccinea W.W.Thomas *

Piperaceae

Peperomia epipremnifolia D.Monteiro \& Leitman

Peperomia riparia Yunck.

Peperomia serpentarioides Miq.

Peperomia sulbahiensis D.Monteiro \& M.Coelho

Piper bahianum Yunck.

Piper robustipedunculum Yunck.

Piper vellosoi Yunck.

Poaceae

Alvimia auriculata Soderstr. \& Londoño *

Alvimia gracilis Soderstr. \& Londoño *

Anomochloa marantoidea Brongn. *

Arberella bahiensis Soderstr. \& Zuloaga *

Atractantha cardinalis Judz. *

Atractantha radiata McClure*

Chusquea clemirae A.C.Mota, R.P.Oliveira \& L.G.Clark

Diandrolyra pygmaea Soderstr. \& Zuloaga ex R.P.Oliveira \& L.G.Clark

Digitaria doellii Mez *

Eremetis robusta Hollowell, F.M.Ferreira \& R.P.Oliveira Eremocaulon aureofimbriatum Soderstr. \& Londoño * Ichnanthus longhi-wagnerae A.C.Mota \& R.P.Oliveira Merostachys annulifera Send.

Merostachys argentea Send.

Merostachys lanata Send.

Merostachys magnispicula Send.

Merostachys medullosa Send.

Merostachys ramosissima Send.

Olyra bahiensis R.P.Oliveira \& Longhi-Wagner

Olyra filiformis Trin.

Olyra latispicula Soderstr. \& Zuloaga*

Parianella carvalhoi (R.P.Oliveira \& Longhi-Wagner)

F.M.Ferreira \& R.P. Oliveira

Parianella lanceolata (Trin.) F.M.Ferreira \& R.P.Oliveira

Paspalum restingense Renvoize *

Paspalum strigosum Döll

Piresia palmula M.L.S.Carvalho \& R.P.Oliveira

Raddia distichophylla (Schrad. ex Nees) Chase *

Raddia stolonifera R.P.Oliveira \& Longhi-Wagner 
Continuation Table 1.

\begin{tabular}{|c|}
\hline Polygalaceae \\
\hline Caamembeca martinelli (Marques \& E.F.Guim.) J.F.B.Pastore * \\
\hline $\begin{array}{l}\text { Caamembeca martinelli var. carnosa (Marques \& E.F.Guim.) } \\
\text { J.F.B.Pastore }\end{array}$ \\
\hline Securidaca revoluta (A.W.Benn.) Marques \\
\hline Primulaceae \\
\hline Cybianthus nemoralis (Mez) G.Agostini \\
\hline Rubiaceae \\
\hline Chomelia bahiae J.H.Kirkbr. \\
\hline $\begin{array}{l}\text { Denscantia andrei (E.L.Cabral \& Bacigalupo) E.L.Cabral \& } \\
\text { Bacigalupo * }\end{array}$ \\
\hline $\begin{array}{l}\text { Denscantia macrobracteata (E.L.Cabral \& Bacigalupo) E.L.Cabral } \\
\& \text { Bacigalupo * }\end{array}$ \\
\hline Faramea bicolor J.G.Jardim \& Zappi \\
\hline Faramea nocturna J.G.Jardim \& Zappi \\
\hline Ixora bahiensis Benth. \\
\hline Ixora cabraliensis Di Maio et Peixoto \\
\hline Malanea harleyi J.H.Kirkbr. * \\
\hline Mitracarpus anthospermoides K.Schum. \\
\hline Posoqueria bahiensis Macias \& Kin.-Gouv. \\
\hline Psychotria martiusii Müll.Arg. \\
\hline Psychotria megalocalyx Müll.Arg. \\
\hline Psychotria salzmanniana Müll.Arg. \\
\hline Psychotria silvicola Müll.Arg. \\
\hline Psychotria strigosa Müll.Arg. \\
\hline Psychotria wawrana Müll.Arg. \\
\hline Rudgea hileiabaiana Zappi \& Bruniera \\
\hline Rudgea ilheotica Müll.Arg. \\
\hline Rudgea involucrata Müll.Arg. \\
\hline Rudgea malpighiacea Standl. \\
\hline Rudgea mouririoides Standl. \\
\hline Salzmannia arborea J.G. Jardim \\
\hline Sphinctanthus insignis Steyerm. \\
\hline Rutaceae \\
\hline Andreadoxa flava Kallunki * \\
\hline Conchocarpus concinnus Kallunki * \\
\hline Conchocarpus dasyanthus Kallunki * \\
\hline Conchocarpus fissicalyx Pirani * \\
\hline Conchocarpus gaudichaudianus subsp. bahiensis Kallunki * \\
\hline Conchocarpus hirsutus Pirani * \\
\hline Conchocarpus inopinatus Pirani * \\
\hline Conchocarpus longipes Kallunki * \\
\hline Conchocarpus mastigophorus Kallunki * \\
\hline Conchocarpus modestus Kallunki* \\
\hline Conchocarpus punctatus Kallunki * \\
\hline Conchocarpus racemosus (Nees \& Mart.) Kallunki \& Pirani \\
\hline
\end{tabular}

Continuation Table 1.

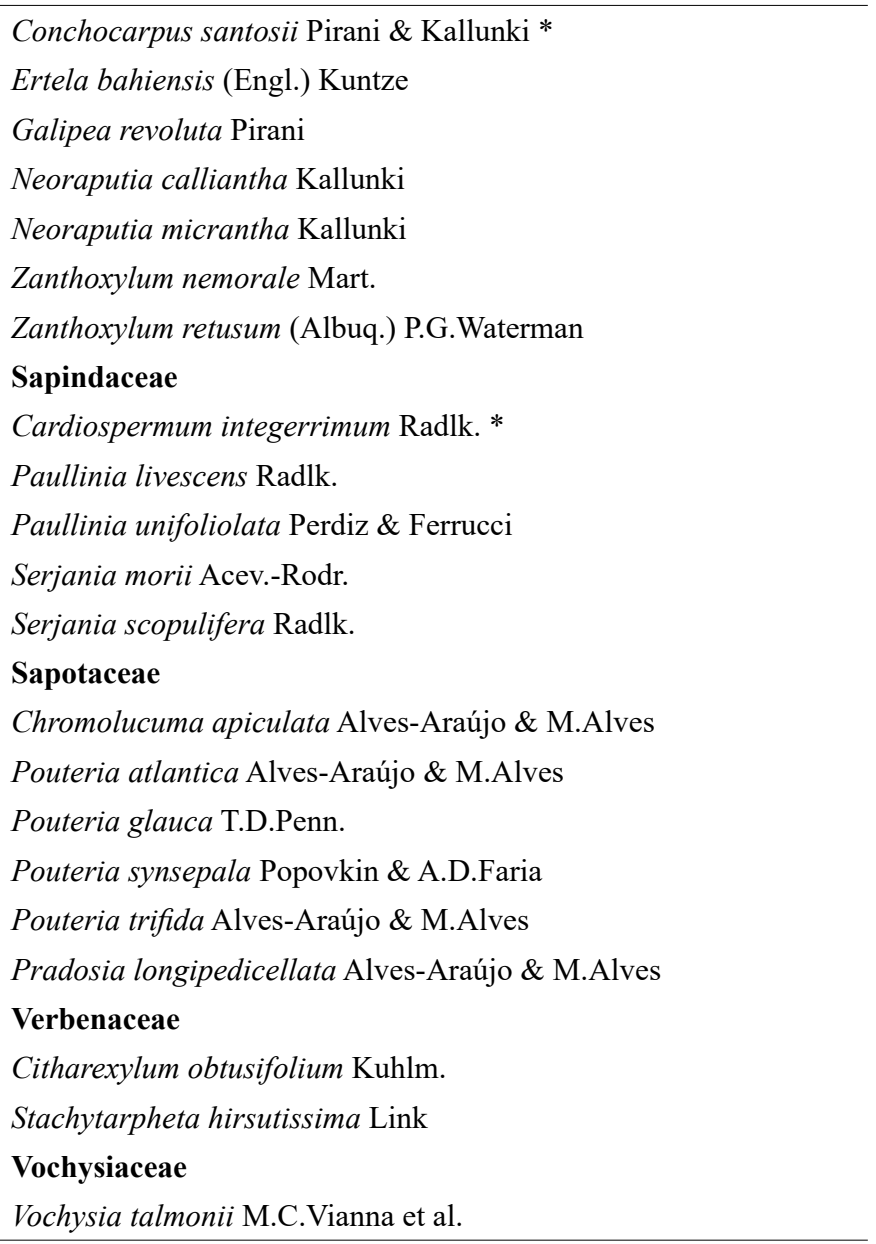

The occurrence of only 12 endemic species of Conchocarpus in the present checklist probably indicates that several endemic species of this genus are exclusively found at more inland seasonally dry forests (Kallunki \& Pirani 1998).

Among the endemic genera from southern Bahia and northern Espírito Santo reported by Thomas et al. (2003), such as Arapatiella and Brodriguesia (Fabaceae), Atractantha, Alvimia and Sucrea (Poaceae), and Trigoniodendron (Trigoniaceae), several are not endemic to BCF. For example, Brodriguesia, Atractantha and Trigoniodendron have been recorded outside our study area, respectively, in Sergipe, Amazonas, and near the Doce River. Similarly, Physeterostemon R.Goldenb. \& Amorim (Melastomataceae), a recently described endemic genus from the Atlantic Forest of Bahia state (Amorim et al. 2014, Goldenberg et al. 2016, Goldenberg \& Amorim 2006), was not considered endemic to $\mathrm{BCF}$ in this checklist, as among its species $P$. gomesii Amorim \& R.Goldenb. occurs in the municipality of Boa Nova (Amorim et al. 2014), which lies slightly outside our study area.

In comparison with the preliminary list of Thomas et al. (2003), which included 395 endemic angiosperm taxa from an area that covered a larger part of northern Espírito Santo state, as far as the Doce River, and the southern Bahia Atlantic Forest, including more inland areas, and almost reaching the municipality of Salvador (i.e., an area ca. $20 \%$ larger than ours) (Figure 1a), the list presented here surprisingly 

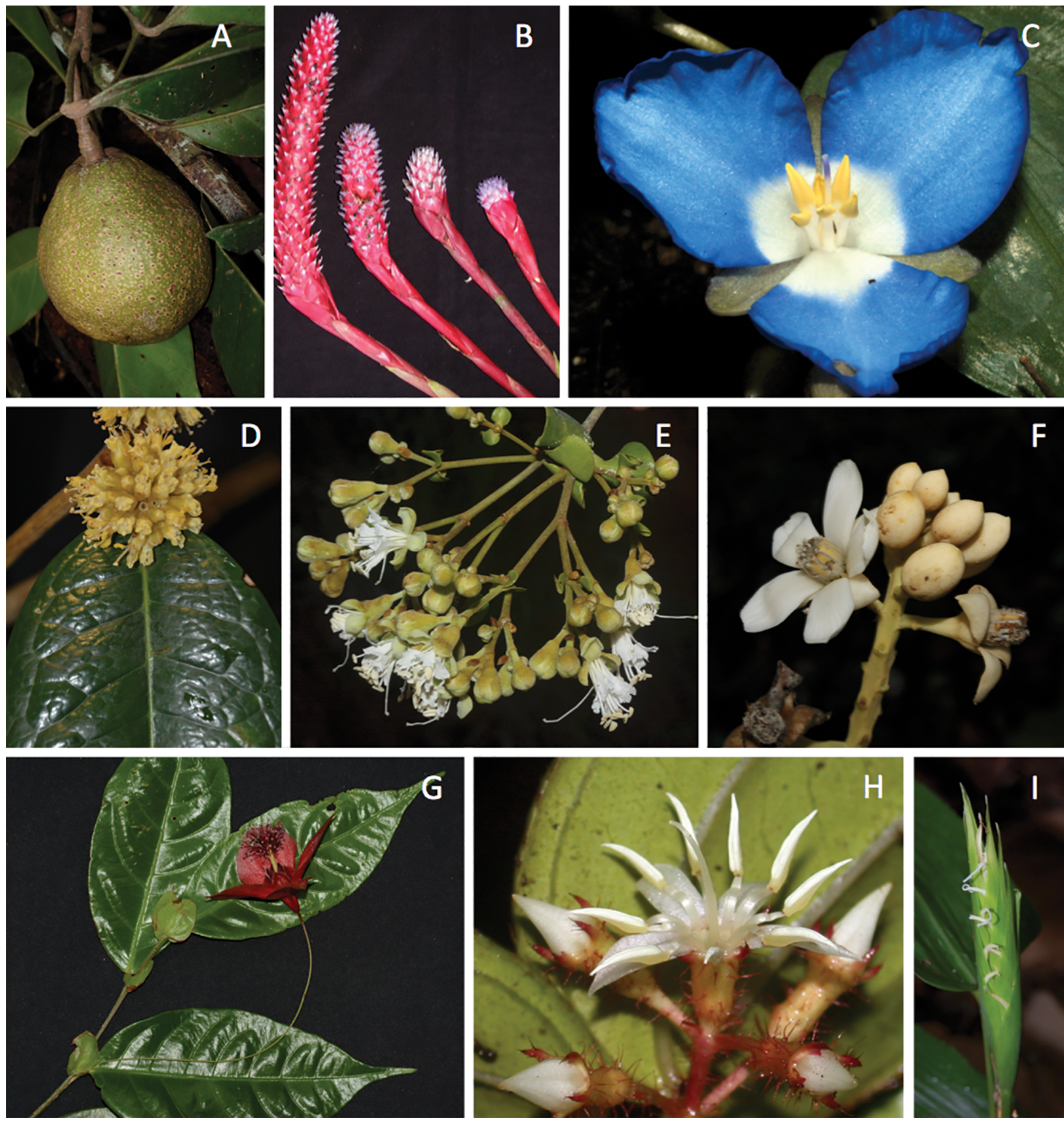

Figure 2. Some species found in the checklist of endemic angiosperms of BCF: a) Kuhlmanniodendron macrocarpum (Achariaceae). b) Quesnelia koltesii (Bromeliaceae). c) Dichorisandra leucophtalmos (Commelinaceae). d) Tapura zei-limae (Dichapetalaceae). e) Arapatiella psilophylla (Fabaceae). f) Harleyodendron unifoliolatum (Fabaceae). g) Pavonia goetheoides (Malvaceae). h) Pleiochiton amorimii (Melastomataceae). i) Anomochloa marantoidea (Poaceae). Photos: a, b, d, g, h) A.Amorim; c) L.Aona; e, f) D.Cardoso; i) J.Jardim.

shares only 143 taxa (Table 1). If we had used the Doce River as our area southern limit, it would have included at least 45 additional species restricted to the Linhares region, such as Cryptanthus beuckeri E.Morren (Bromeliaceae), Rourea luizalbertoi Forero, L.A.Vidal \& Carbonó (Connaraceae), Simira eliezeriana Peixoto (Rubiaceae) and the endemic genus Riodocea Delprete (Thomas et al. 2003, Rolim et al. 2016). Despite being geographically close and having several species in common (Saiter et al. 2016b), the vegetation of Linhares and the
Doce River floodplain are ecologically different from the BCF (Rolim et al. 2005, 2006). In floristic terms, the Linhares region may be more similar to northern Rio de Janeiro, southern Espírito Santo (Silva \& Nascimento 2001, Saiter et al. 2016b) and eastern Minas Gerais states (see Krenák-Waitaká Forests sensu Saiter et al. 2016a).

Another difference between the two checklists is due to the time gap of about 15 years between studies. A total of 174 species in our checklist were described after 2003, probably due to increased efforts 
in collecting and studying plants in the Atlantic Forest in Bahia. For instance, about $41.5 \%$ of all new angiosperms in the Brazilian flora described between 1990 and 2006 are endemic to the Atlantic Forest (Sobral \& Stehmann 2009). Moreover, 23 species of angiosperms endemic to BCF were described between 2015 and 2017 (e.g., Araújo et al. 2015, Santos et al. 2015, Abreu \& Giulietti 2016, Amorim et al. 2016, Côrtes et al. 2016, Ferreira et al. 2016, Marinho \& Lucas 2016, Popovkin et al. 2016, Ribeiro et al. 2016, Terra-Araujo et al. 2016, Daly \& Melo 2017, Gonçalez et al. 2017).

Compared to the results of a search in the Brazilian Plant List (Flora do Brasil 2020, under construction) using the following filters: "group: angiosperms", "endemism: only endemic to Brazil", "occurrence: only occurs in", "origin: native", "state: Bahia", and "phytogeographic domain: Atlantic Forest", the number of species in our checklist (547 spp.) was much smaller than the 795 species identified by the search engine. Among the reasons for this large difference ( $248 \mathrm{spp}$.) it is worth mentioning that a) more than 50 taxa from our list had to be removed due to lack of precise geospatial data, such as the municipality name, which precluded us from citing them as endemic to BCF; b) among the taxa exclusive to the Brazilian Plant List (Flora do Brasil 2020, under construction) that are not restricted to $\mathrm{BCF}$, several occur in more inland seasonally dry forests in the Atlantic Forest, such as Chrysophyllum subspinosum Monach. (Sapotaceae) and Colicodendron bahianum Cornejo \& Iltis (Capparaceae); and c) at least 29 species exclusive to the Brazilian Plant List search occur close to, but outside the study area, such as Canistrum camacaense Martinelli \& Leme (Bromeliaceae), Inga grazielae (Vinha) T.D.Penn. (Fabaceae), and Bertolonia carmoi Baumgratz (Melastomataceae), all from the municipality of Boa Nova, and Aechmea bicolor L.B.Sm. (Bromeliaceae), Heteropterys jardimii Amorim (Malpighiaceae), and Passiflora timboensis T.S.Nunes \& L.P.Queiroz (Passifloraceae), from the municipality of Santa Teresinha.

There are additional reasons to anticipate that the difference in species numbers between the Brazilian Plant List and our list could be even greater, as the list presented here includes 84 taxa that also occur in northern Espírito Santo and in a small area in northeastern Minas Gerais (Figure 1c). Moreover, the Brazilian Plant List (Flora do Brasil 2020, under construction) is an ongoing project on which new taxa can be continuously added and identified as endemic, what could bring the total number of endemic angiosperm species using the same filters even larger. Alternatively, if the native distribution of several taxa is shown to extend beyond the Atlantic Forest domain in Bahia, the difference between the lists could decrease, as fewer species from the Brazilian Plant List would appear as endemic while applying the same search filters.

Developing knowledge on patterns of endemism for plant species is extremely important and must be taken into account in conservation strategies. This information is relevant for the establishment of new protected areas (Kerr 1997), restoration policies (Chazdon 2008) and in directing additional studies (Francisco-Ortega et al. 2010). Considering the key role of endemic species in the design of conservation policies (Myers et al. 2000, Van Der Werff \& Consiglio 2004, Moraes et al. 2005, Lamoreux et al. 2006, Martinelli et al. 2008, Essl et al. 2009, Nowak \& Nobis 2010), we expect this checklist could guide future studies and conservation strategies, as well as emphasize the importance of the BCF region as a key area for plant conservation.

\section{Supplementary material}

The following online material is available for this article:

Appendix 1-Taxonomic studies and specialists consulted by family.

\section{Acknowledgements}

The authors thank Coordenação de Aperfeiçoamento de Pessoal de Nível Superior (CAPES) for awarding a Master Fellowship to POB, Conselho Nacional de Desenvolvimento Científico e Tecnológico (CNPq) for the Research Productivity Fellowship to AMA (grant \# 310717/2015-9), and PF (grant \# 306228/2016-5), and all specialists that provided updated information on endemic species distribution.

\section{Author Contributions}

Piera Ostroski: substantial contribution in the concept and design of the study; contribution to data collection; contribution to data analysis and interpretation; contribution to manuscript preparation; contribution to critical revision augmenting intellectual content.

Felipe Saiter: substantial contribution in the concept and design of the study; contribution to data analysis and interpretation; contribution to manuscript preparation; contribution to critical revision augmenting intellectual content.

André Amorim: substantial contribution in the concept and design of the study; contribution to data analysis and interpretation; contribution to manuscript preparation; contribution to critical revision augmenting intellectual content.

Pedro Fiaschi: substantial contribution in the concept and design of the study; contribution to data analysis and interpretation; contribution to manuscript preparation; contribution to critical revision augmenting intellectual content.

\section{Conflicts of interest}

The authors declare that there is no conflict of interest related to the publication of the data in this article.

\section{References}

ABREU, I.S. \& GIULIETTI, A.M. 2016. Aristolochia brunneomaculata, a new threatened species of Aristolochiaceae from the Atlantic Forest in Bahia, Brazil. Sitientibus 16:10.13102/scb1060.

AMORIM, A.M., JARDIM, J.G. \& GOLDENBERG, R. 2014. Physeterostemon gomesii (Melastomataceae): the fourth species of this endemic genus in Bahia, Brazil. Phytotaxa 175(1):45-50.

AMORIM, A.M., LISBOA, D.S., MARINHO, L.C. \& FIASCHI, P. 2016. Novelties in Tapura (Dichapetalaceae) from the Brazilian Atlantic Forest. Syst. Bot. 41(3):747-757.

ARAÚJO, T., FIASCHI, P. \& AMORIM, A.M. 2015. Erythroxylum riparium (Erythroxylaceae), a new species from the Brazilian Atlantic Forest. Phytotaxa 230(1):75-80.

BERRY, P.E., HAHN, W.J., SYTSMA, K.J., HALL, J.C. \& MAST, A. 2004. Phylogenetic relationships and biogeography of Fuchsia (Onagraceae) based on noncoding nuclear and chloroplast DNA data. Am. J. Bot. 91(4):601-614.

BFG. 2015. Growing knowledge: An overview of Seed Plant diversity in Brazil. Rodriguésia 66(4):1085-1113.

CARNAVAL, A.C., HICKERSON, M.J., HADDAD, C.F.B., RODRIGUES, M.T. \& MORITZ, C. 2009. Stability predicts genetic diversity in the Brazilian Atlantic forest hotspot. Science 323(5915):785-789. 
CHAZDON, R.L. 2008. Beyond Deforestation: Restoring Forests and Ecosystem Services on Degraded Lands. Science 320(5882):1458-1460.

COLOMBI, V.H., LOPES, S.R. \& FAGUNDES, V. 2010. Testing the Rio Doce as a riverine barrier in shaping the atlantic rainforest population divergence in the rodent Akodon cursor. Genet. Mol. Biol. 33(4):785-789.

CÔRTES, A.L.A., DANIEL, T.F. \& RAPINI, A. 2016. Taxonomic revision of the genus Schaueria (Acanthaceae). Plant Syst. Evol. 302:819-851.

CRACRAFT, J. 1985. Historical Biogeography and Patterns of Differentiation within the South American Avifauna : Areas of Endemism. Ornithol. Monogr. 36:49-84.

DALY, D.C. \& MELO, M. da F. 2017. Four new species of Trattinnickia from South America. Studies in Neotropical Burseraceae XXII. Brittonia 69(3):376-386.

ESSL, F., STAUDINGER, M., STÖHR, O., SCHRATT-EHRENDORFER, L., RABITSCH, W. \& NIKLFELD, H. 2009. Distribution patterns, range size and niche breadth of Austrian endemic plants. Biol. Conserv. 142(11):2547-2558

FERREIRA, F.M., HOLLOWELL, V.C. \& OLIVEIRA, R.P. 2016. Eremitis linearifolia and E. robusta (Poaceae, Bambusoideae, Olyreae): two new species of herbaceous bamboos from Brazil first collected over 30 years ago. Phytotaxa 280(2):179-189.

FLORA DO BRASIL 2020 under construction. Jardim Botânico do Rio de Janeiro. http://floradobrasil.jbrj.gov.br/ (acessed on 09/Mar/2017)

FRANCISCO-ORTEGA, J., WANG, F.G., WANG, Z.S., XING, F.W., LIU, H., XU, H., XU, W.X., LUO, Y.B., SONG, X.Q., GALE, S., BOUFFORD, D.E., MAUNDER, M. \& AN, S.Q. 2010. Endemic Seed Plant Species from Hainan Island: A Checklist. Bot. Rev. 76(3):295-345.

FUNDAÇÃO SOS MATA ATLÂNTICA. 2013. Atlas dos remanescentes florestais da Mata Atlântica Período 2011-2012.

FUNDAÇÃO SOS MATA ATLÂNTICA. 2014. Atlas dos remanescentes florestais da Mata Atlântica Período 2012-2013.

GOLDENBERG, R. \& AMORIM, A.M. 2006. Physeterostemon (Melastomataceae): a new genus and two new species from the Bahian Atlantic Forest, Brazil. Taxon 55(4):965-972.

GOLDENBERG, R., MICHELANGELI, F.A., AONA, L.Y.S. \& AMORIM, A.M. 2016. Angiosperms and the Linnean shortfall: three new species from three lineages of Melastomataceae at one spot at the Atlantic Forest. PeerJ 4:1824-1833.

GONÇALEZ, V.M., PFEIL, B.E., ANTONELLI, A. \& DUARTE, M.C. 2017 Two new species of Pavonia (Malvoideae, Malvaceae) from southern Bahia, Brazil. Phytotaxa 305(2):97-103.

IBGE. 1993. Mapa de Vegetação do Brasil. 2nd ed. Diretoria de Geociências, Rio de Janeiro.

KALLUNKI, J.A. \& PIRANI, J.R. 1998. Synopses of Angostura Roem. \& Schult. and Conchocarpus J. C. Mikan (Rutaceae). Kew Bull. 53(2):257-334.

KERR, J.T. 1997. Species Richness, Endemism, and the Choice of Areas for Conservation. 11(5):1094-1100.

LAMOREUX, J.F., MORRISON, J.C., RICKETTS, T.H., OLSON, D.M., DINERSTEIN, E., MCKNIGHT, M.W. \& SHUGART, H.H. 2006. Global tests of biodiversity concordance and the importance of endemism. Nature 440(7081):212-214.

MARINHO, L.C. \& LUCAS, E. 2016. ANew Species of Myrcia sect. Aulomyrcia (Myrtaceae) from the restingas of Bahia, Brazil. Phytotaxa 280(3):285-291.

MARTINELLI, G., VIEIRA, C., GONZALEZ, M., LEITMAN, P.M., PIRATININGA, A., COSTA, A.F. \& FORZZA, R.C. 2008. Bromeliaceae da Mata Atlântica Brasileira: lista de espécies, distribuição e conservação. Rodriguésia 59(1):209-258.

MARTINI, A.M.Z., FIASCHI, P., AMORIM, A.M. \& PAIXÃO, J.L. 2007. A hot-point within a hot-spot: A high diversity site in Brazil's Atlantic Forest. Biodivers. Conserv. 16(11):3111-3128.

MARTINS, F.M. 2011. Historical biogeography of the Brazilian Atlantic forest and the Carnaval-Moritz model of Pleistocene refugia: What do phylogeographical studies tell us? Biol. J. Linn. Soc. 104(3):499-509.
MENINI NETO, L., FURTADO, S.G., ZAPPI, D.C., OLIVEIRA FILHO, A.T. \& FORZZA, R.C. 2016. Biogeography of epiphytic Angiosperms in the Brazilian Atlantic forest, a world biodiversity hotspot. Rev. Bras. Bot. 39(1):261-273.

MURRAY-SMITH, C., BRUMMITT, N.A., OLIVEIRA-FILHO, A.T., BACHMAN, S., MOAT, J., LUGHADHA, E.M.N. \& LUCAS, E.J. 2009. Plant diversity hotspots in the Atlantic coastal forests of Brazil. Conserv. Biol. 23(1):151-163.

MYERS, N., MITTERMEIER, R.A., MITTERMEIER, C.G., FONSECA, G.A.B. \& KENT, J. 2000. Biodiversity hotspots for conservation priorities Nature 403(6772):853-858.

NOWAK, A. \& NOBIS, M. 2010. Tentative list of endemic vascular plants of the Zeravshan Mts in Tajikistan: distribution, habitat preferences and conservation status of species. Biodivers. Res. Conserv. 19:65-80.

OLIVEIRA-FILHO, A.T., TAMEIRÃO-NETO, E., CARVALHO, W.A.C., WERNECK, M., BRINA, A.E., VIDAL, C. V, REZENDE, S.C. \& PEREIRA, J.A.A. 2005. Análise florística do compartimento arbóreo de áreas de floresta atlântica sensu lato na região das Bacias do Leste (Bahia, Minas Gerais, Espírito Santo e Rio de Janeiro). Rodriguésia 56(87):185-235.

OLIVEIRA-FILHO, A. \& FONTES, M. 2000. Patterns of Floristic Differentiation among Atlantic Forests in Southeastern Brazil and the Influence of Climate. Biotropica 32(4b):793-810.

POPOVKIN, A. V, FARIA, A.D.D.E. \& SWENSON, U.L.F. 2016. Pouteria synsepala (Sapotaceae: Chrysophylloideae): a new species from the northern littoral of Bahia, Brazil. Phytotaxa 286(1):39-46.

QUANTUM GIS DEVELOPMENT TEAM. 2016. Quantum GIS Geographic Information System. Open Source Geospatial Found. Proj.

RIBEIRO, M., MORI, S.A., ALVES-ARAÚJO, A. \& PEIXOTO, A.L. 2016. A new species of Eschweilera (Lecythidaceae) from the Brazilian Atlantic Forest. Phytotaxa 255(3):267-273.

ROLIM, S.G., JESUS, R.M., NASCIMENTO, H.E.M., DO COUTO, H.T.Z. \& CHAMBERS, J.Q. 2005. Biomass change in an Atlantic tropical moist forest: The ENSO effect in permanent sample plots over a 22 -year period. Oecologia 142(2):238-246.

ROLIM, S.G., IVANAUSKAS, N.M., RODRIGUES, R.R., NASCIMENTO, M.T., GOMES, J.M.L., FOLLI, D.A. \& COUTO, H.T.Z. do. 2006. Composição Florística do estrato arbóreo da Floresta Estacional Semidecidual na Planície Aluvial do rio Doce, Linhares, ES, Brasil. Acta Bot. Brasilica 20(3):549-561.

ROLIM, S.G., MAGNAGO, L.F.S., SAITER, F.Z., AMORIM, A.M. \& ABREU, K.M.P. de. 2016. São as florestas do norte do espírito santo e sul da bahia as mais ricas em espécies arbóreas no domínio da floresta atlântica? In Floresta Atlântica de Tabuleiro: diversidade e endemismo na Reserva Natural Vale (A. C. Rolim, Samil Gonçalves; Menezes, Luis F. T. de; Srbek-Araujo, ed.). Editora Rona, Belo Horizonte, p.91-100.

SAITER, F.Z., BROWN, J.L., THOMAS, W.W., DE OLIVEIRA-FILHO, A.T. \& CARNAVAL, A.C. 2016a. Environmental correlates of floristic regions and plant turnover in the Atlantic Forest hotspot. J. Biogeogr. 43(12):2322-2331.

SAITER, F.Z., ROLIM, S.G. \& DE OLIVEIRA-FILHO, A.T. 2016b. A floresta de Linhares no contexto fitogeográfico do leste do Brasil. In Floresta Attântica de Tabuleiro: diversidade e endemismo na Reserva Natural Vale (A. C. Rolim, Samil Gonçalves; Menezes, Luis F. T. de; Srbek-Araujo, ed.). Editora Rona, Belo Horizonte, p.61-69.

SANTOS, M.F., LUCAS, E., SOBRAL, M. \& SANO, P.T. 2015. New species of Myrcia s.l. (Myrtaceae) from Campo Rupestre, Atlantic Forest and Amazon Forest. Phytotaxa 222(2):100-110.

SANTOS, M.M., CAVALCANTI, D.R., SILVA, J.M.C. da \& TABARELLI, M. 2007. Biogeographical relationships among tropical forests in north-eastern Brazil. J. Biogeogr. 34(3):437-446.

SILVA, G.C. da \& NASCIMENTO, M.T. 2001. Fitossociologia de um remanescente de mata sobre tabuleiros no norte do estado do Rio de Janeiro (Mata do Carvão). Rev. Bras. Botânica 24(1):51-62.

SILVA, J.M.C., SOUSA, M.C. \& CASTELLETTI, C.H.M. 2004. Areas of endemism for passerine birds in the Atlantic forest, South America. Glob. Ecol. Biogeogr. 13:85-92. 
Ostroski, P. et al.

SOBRAL, M. \& STEHMANN, J.R. 2009. An analysis of new angiosperm species discoveries in Brazil (1990-2006). Taxon 58(1):227-232.

TERRA-ARAUJO, M.H., FARIA, A.D. \& SWENSON, U. 2016. A taxonomic update of Neotropical Pradosia (Sapotaceae, Chrysophylloideae). Syst. Bot. 41(3):634-650.

THOMAS, W.W., CARVALHO, A.M. V, AMORIM, A.M., GARRISON, J. \& ARBELAÉZ, A.L. 1998. Plant endemism in two forests in southern Bahia, Brazil. Biodivers. Conserv. 7:311-322.

THOMAS, W.W., JARDIM, J.G., FIASCHI, P. \& AMORIM, A.M. 2003. Lista preliminar das Angiospermas localmente endêmicas do Sul da Bahia e Norte do Espírito Santo, Brasil. In Corredor de Biodiversidade da Mata Atlântica do Sul da Bahia (PRADO, P.I., LANDAU, E.C., MOURA, R.T., PINTO, L.P.S., FONSECA, G.A.B. \& ALGER, K. orgs.). Published in CD-ROM, Ilhéus, IESB/ CI/ CABS/ UFMG/ UNICAMP.
VAN DER WERFF, H. \& CONSIGLIO, T. 2004. Distribution and conservation significance of endemic species of flowering plants in Peru. Biodivers. Conserv. 13(9):1699-1713.

WHITTAKER, R.J., ARAÚJO, M.B., JEPSON, P., LADLE, R.J., WATSON, J.E.M. \& WILLIS, K.J. 2005. Conservation biogeography: Assessment and prospect. Divers. Distrib. 11(1):3-23.

Received: $29 / 03 / 2018$

Revised: $11 / 07 / 2018$

Accepted: $12 / 07 / 2018$

Published online: 16/08/2018 